\title{
Primary Cervical Intradural Extramedullary Primitive Neuroectodermal Tumors Presented as Acute Transverse Myelitis: A Case Report and Review of Literature
}

\author{
Partha Ghosh ${ }^{1}$ Kaushik Roy ${ }^{1}$ Sneha J. Bothra ${ }^{1}$ Sandeep B.V ${ }^{1}$ \\ ${ }^{1}$ Department of Neurosurgery, Nil Ratan Sircar Medical College and \\ Hospital, Kolkata, West Bengal, India \\ Indian J Neurosurg 2017;6:67-71. \\ Address for correspondence Dr. Partha Ghosh, MBBS, MS, \\ Department of Neurosurgery, Nil Ratan Sircar Medical College and \\ Hospital, 5th Floor, UNB Building, 138 A.J.C Bose Road, Kolkata, West \\ Bengal 700014, India (e-mail: ghoshparthanrs@gmail.com).
}

\begin{abstract}
Keywords

- primitive neuroectodermal tumor

- intradural extramedullary spinal tumor

- cervical spinal cord

Primary spinal cord presentation of primitive neuroectodermal tumors (PNET) is a rare occurrence and carries a poor prognosis. A 20 -year-old female patient presented with pain in the neck region, bilateral upper and lower limb weakness, and bladder and bowel dysfunction. Neurological examination revealed the marked paresis with hyporeflexia of both the upper and hyperreflexia of lower extremities. Preoperative magnetic resonance imaging of the spine revealed an intradural mass at the C6-D1 level. A laminectomy of C5 to upper half D1 vertebra level and near-total removal of the tumor was performed. Pathological findings were consistent with PNET. The clinical, histopathological, and radiological findings of the patient are presented.
\end{abstract}

\section{Introduction}

Extracranial primitive neuroectodermal tumor (PNET) originates from the soft tissues or bones outside the central or the sympathetic nervous system and are composed of differentiated small, round, and hyperchromatic tumor cells. ${ }^{1}$

Spinal PNETs are most common in adults, with a male predominance. ${ }^{2}$ These PNETs have a tendency to metastasize outside neuroaxis with the most frequent sites being the lungs, bones, and lymph nodes, similar to intracranial PNETs. ${ }^{3}$ The aggressive nature of the tumor is highlighted by its rapid recurrence rate. ${ }^{4}$

Intraspinal PNET with intradural location is an exceedingly rare entity as reported in the literature ${ }^{5}$ and in adults, the commonest localization is intradural or intramedullary. ${ }^{6}$

Cases of PNET have been increasingly reported in recent years, but there is a scarcity of reports of PNET originating primarily from the spinal cord. To the best of our knowledge, only 83 cases of the whole spine and 8 cases of the cervical spine have been reported in the literature till date. ${ }^{7}$

received

May 12, 2016

accepted

June 20, 2016

published online

November 22, 2016
The usual presentation of spinal PNET is a progressive weakness with radiculopathy but presentation with acuteonset weakness is unusual and only one case report presented with acute-onset neurodeficit was published till date, where the tumor was intramedullary in the location at C5-C7 level. ${ }^{8}$

In our present case report, we describe a case of intradural extramedullary PNET at C6-D1 level with extraspinal extension presented as acute transverse myelitis which is probably the first case with such presentation where the tumor is located in intradural extramedullary (IDEM) compartment.

\section{Case Illustration}

A 20-year-old female patient with no significant medical comorbidity presented to the emergency department of our institution with rapidly progressive ascending weakness and sensory changes started from lower limbs over a period of 2 days followed by acute retention of urine. The patient was promptly taken care at the medical ward, catheterized, and
DOI http://dx.doi.org/

10.1055/s-0036-1588033. ISSN 2277-954X. (c) 2017 Neurological Surgeons' Society of India
License terms

(요 (1) $\Theta \circledast$ 

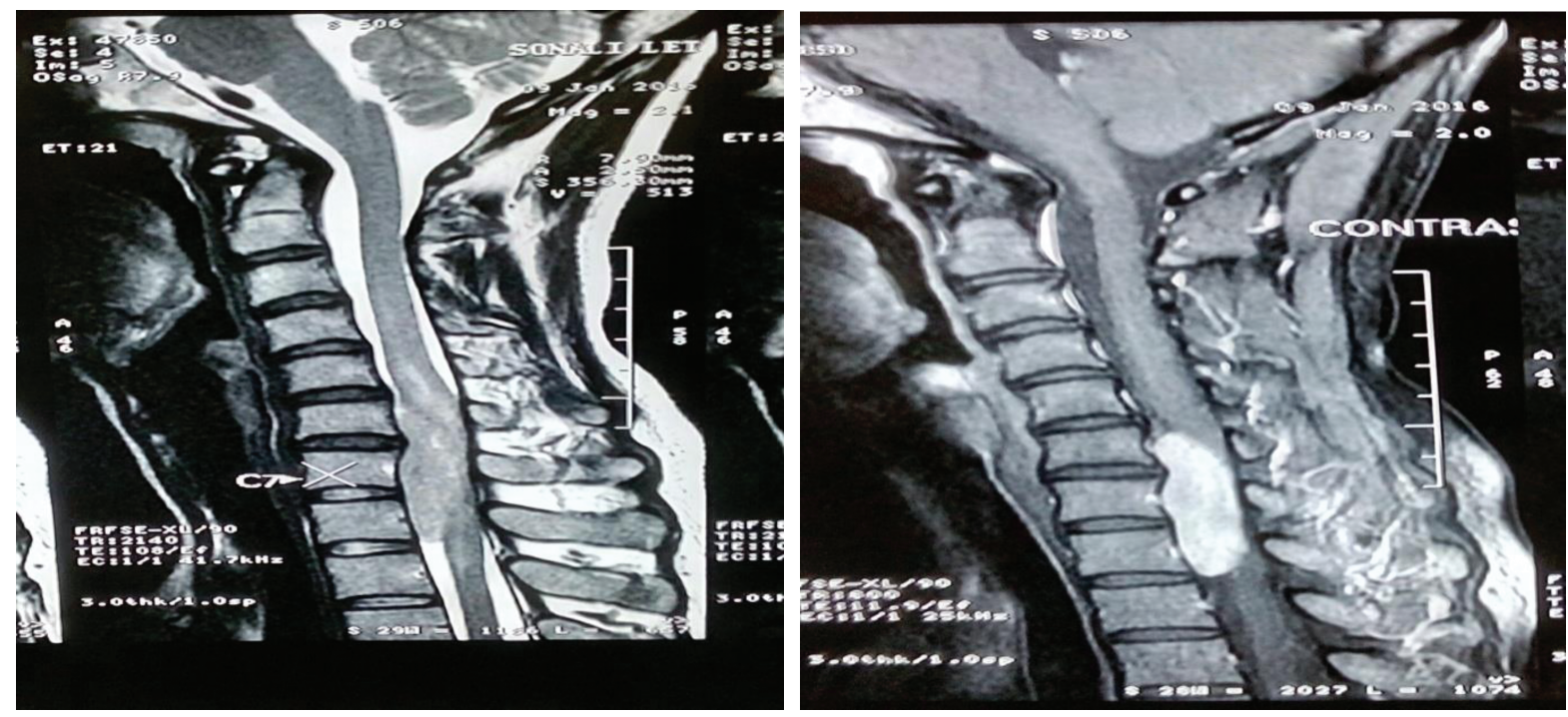

Fig. 1 T2-weighted image of cervical spine showing intradural extramedullary lesion at level of C5, C6, and D1, contrast image shows contrast enhancement.

investigated as a case of acute transverse myelitis. During imaging (magnetic resonance imaging cervical spine) it was evident that patient having an IDEM lesion with an extracanalicular extension at the level of C6, thus referred to get a neurosurgical opinion. On questioning it was revealed that only numbness involving all four limbs and trunk started 1 month before the present episode. She noticed rapid progressive distal to proximal asymmetric weakness only 2 days before admission. Clinically, there was a loss of sensory and motor function below shoulder (below C5), without evidence of any cardiopulmonary compromise. On examination, there was generalized wasting among which right upper limb wasting was significant and the upper limb power is 3/5 with a bilateral weak hand grip, and lower limb power was $0 / 5$. Lower limb deep tendon reflexes were brisk and triceps jerk bilaterally lost, plantar reflexes were equivocal. She was catheterized and constipated. A magnetic resonance image of the cervical spine with contrast, showed fairly large extramedullary lesion involving the anterior and right to the spinal canal extended from C7 to D1 level (- Fig. 1). The lesion showed extension through exiting recess mainly on the right side in the paravertebral portion and in the apical portion of the lung. Above lesion measures approximately $48 \mathrm{~mm} \times 23 \mathrm{~mm} \times 85 \mathrm{~mm}$. The lesion was iso- to hypointense in $\mathrm{T} 1$, iso to hyperintense in $\mathrm{T} 2$, and had heterogeneous contrast enhancement ( $\boldsymbol{F}$ Fig. 2). Spinal cord also showed intramedullary signal changes at the adjacent level. The radiological diagnosis was made as neurofibroma. The patient was prepared for surgery and started on intravenous dexamethasone.

Surgery performed with the aim of spinal decompression and excision of the lesion, through a posterior approach in the prone position. C5, C6, C7, and upper half D1 laminectomy was performed with preservation of facet joints. The dura was tensed and nonpulsatile. Dura opened in the midline and tented laterally; revealing reddish colored soft space occupying lesion (SOL) in the right anterolateral to the cord. The lesion was moderately vascular suckable without a true capsule, adhered to arachnoid and cord. Cord edema and cord changes were visible at that site. Intratumoral decompression of the lesion done, there was intratumoral bleed detected probably this intratumoral bleed was the cause of acute neurological deterioration of the patient. The tumor seems to extend along right $\mathrm{C} 6$ root and through the neural foramina. Surgery kept limited to removal of an intradural portion of the tumor and extraspinal extension removal not attempted. Dural closure and closure of the wound was done in layers following proper hemostasis. The patient was successfully extubated and shifted to the neurosurgical intensive care unit.

Postoperative period was uneventful and patient's neurological status was improved partially. Histopathological analysis of the tumor showed neoplastic lesion composed of small round cells having dark round nuclei and scanty cytoplasm. The cells were separated by fibrous septae. There were areas of necrosis, hemorrhage, and there is an increasing mitotic activity (-Fig. 3). Morphology being suggestive of the PNET. Immunohistological analysis was advised, but could not be done due to financial constraints. Postoperative imaging showed complete removal of an intradural portion of the tumor along with central necrosis of the extraspinal component (-Fig. 4).

\section{Discussion}

\section{History and Classification}

Primary spinal PNETs are histologically indistinguishable from other neural axis PNETs. The earliest recognized case report of a spinal PNET is from an article by Smith et al. ${ }^{9}$ Their use of the term PNET is problematic, however, because the nomenclature and criteria for diagnosing PNET were not formally introduced until 1973 by Hart and Earle, ${ }^{10}$ and therefore it is unclear whether or not the tumor they reported actually met the 

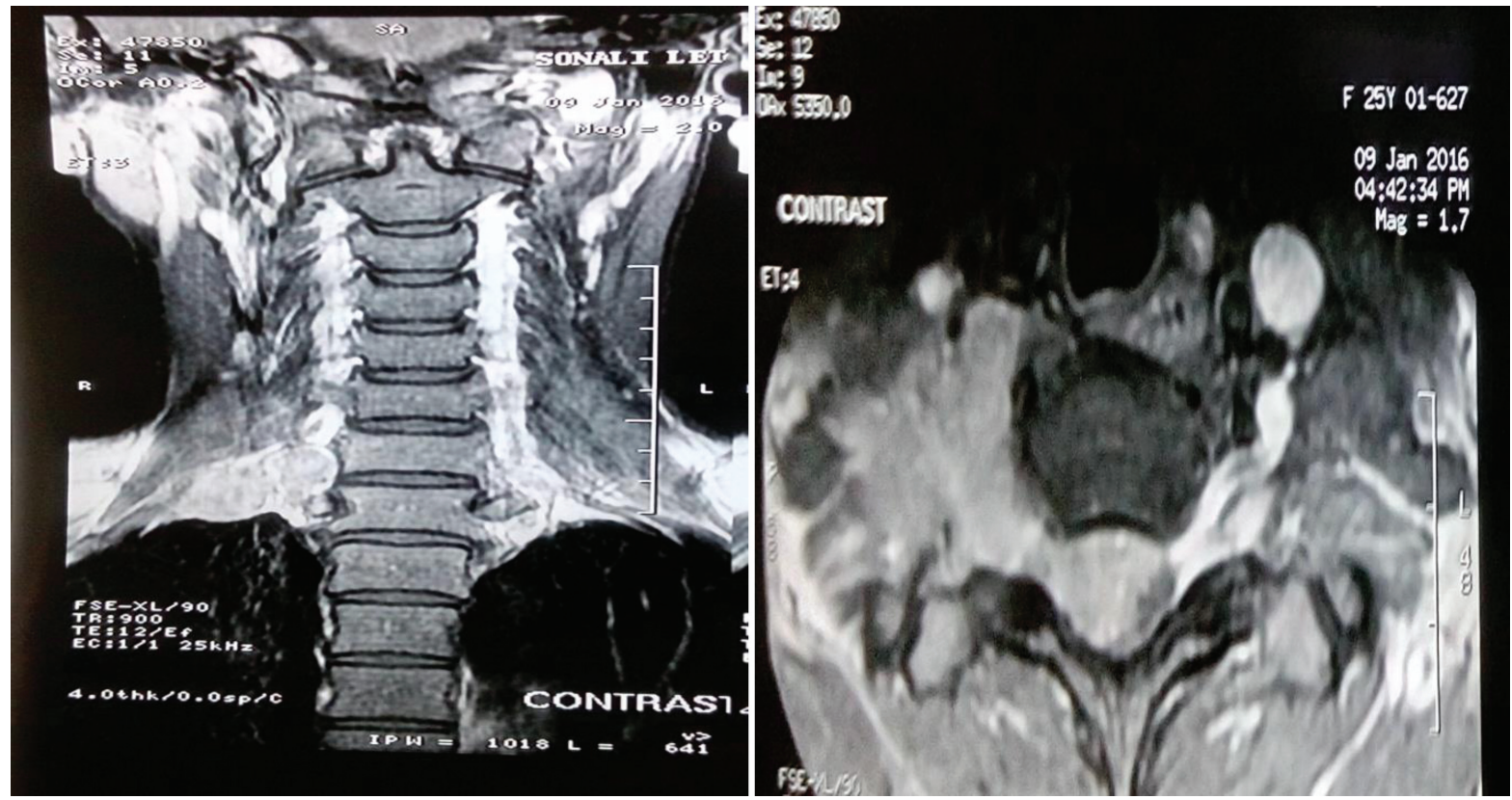

Fig. 2 Contrast coronal image shows right paravertebral extension. Axial cut also shows right paravertebral extension of tumor with contrast enhancement.

criteria for a PNET. The original classification scheme for PNETs arose from the hypothesis that all tumors of this category share a common progenitor cell. The World Health Organization description of PNETs has evolved such that several tumors once included under this nomenclature are now understood as distinct diagnoses, including such notable examples as medulloblastoma, atypical teratoid/rhabdoid tumors, and pineoblastomas. ${ }^{11}$ Similarly, it has been argued that embryonal tumor with abundant neuropil and true rosettes represents a distinct entity as well. ${ }^{12}$

Those tumors currently understood as PNETs are further divided into two categories: central nervous system PNETs

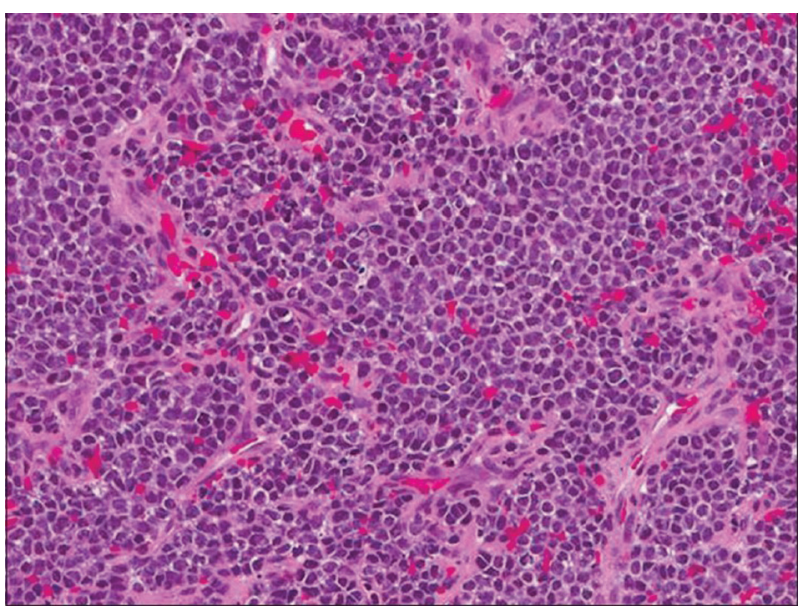

Fig. 3 Neoplastic lesion composed of small round cells having dark round nuclei and scanty cytoplasm. The cells were separated by fibrous septae. and Ewing sarcoma/PNETs. They can both be found anywhere along the neural axis. Genetic and immunohistopathological analyses are used to distinguish these two subtypes, but the clinical significance of this segregation continues to remain unclear.

\section{Epidemiology}

A recent large series of patients indicates that PNETs represent less than $1 \%$ of primary spinal tumors. ${ }^{13}$ The assertion that primary spinal PNETs are most prevalent in the pediatric and young-adult populations and are observed more commonly in males than in females. ${ }^{14}$ In our case, the patient was a 20 -year-old female.

\section{Clinical Presentation}

Clinical presentation of spinal PNETs is variable. The development of pain, paresthesia, weakness, or incontinence over several weeks to months are commonly observed. ${ }^{8}$ Conversely, in our case intratumoral hemorrhage of the intradural tumor resulting in an acute neurological decline, has not been previously reported as per searching of published literature.

\section{Radiographic Findings}

Radiographic findings of spinal PNET vary from patient to patient. Magnetic resonance imaging with Gd contrast is the imaging modality of choice in detecting spinal PNETs. As in our case, PNETs are typically hypo- to isointense on T1 and iso- to hyperintense on $\mathrm{T} 2$, there is often minimal contrast enhancement, but in our case contrast enhancement was homogenous, thus confusing with the preoperative impression of nerve sheath tumor. 

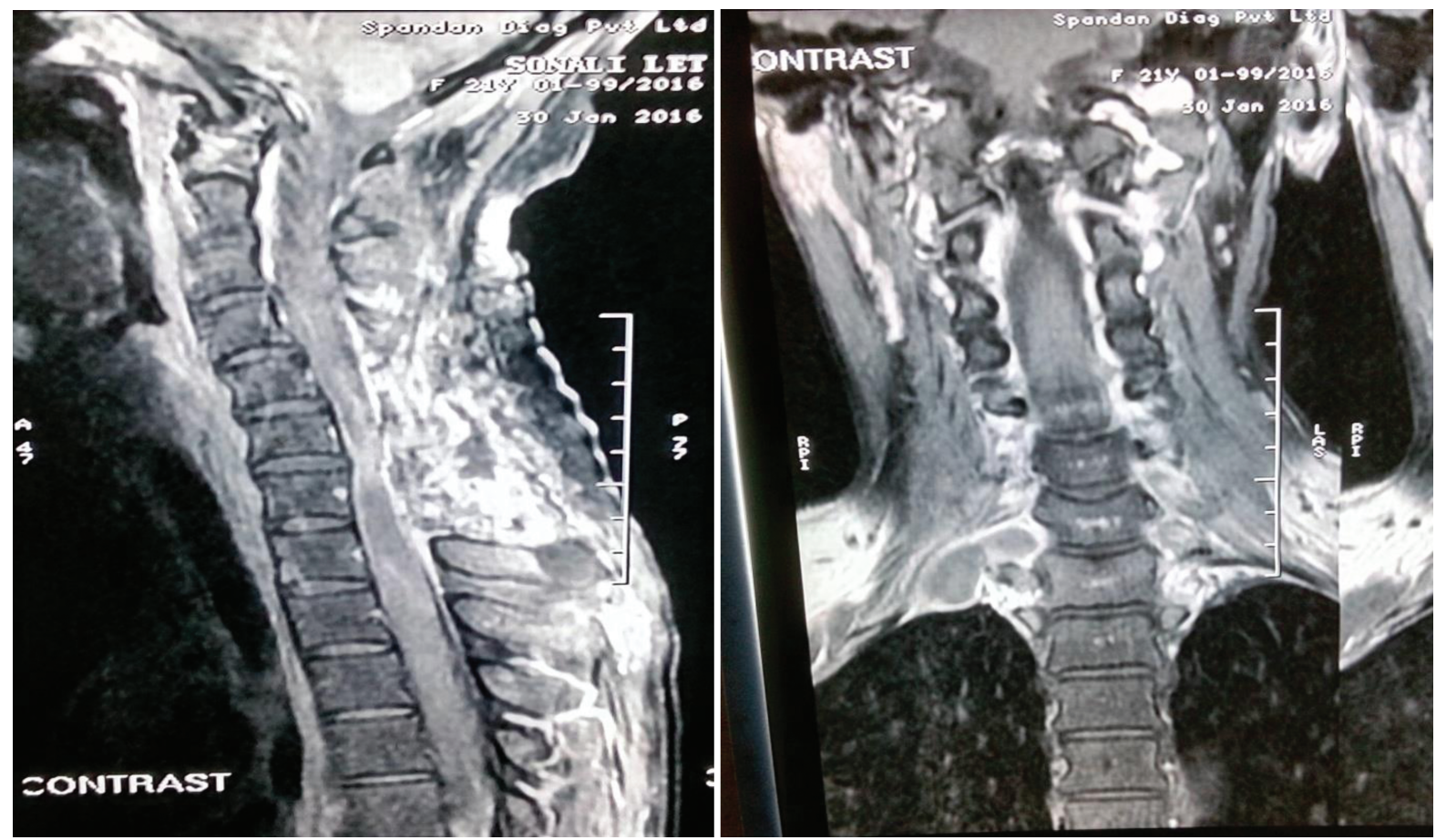

Fig. 4 Postoperative sagittal contrast MRI shows no residual intraspinal tumor but coronal contrast MRI shows residual paravertebral tumor with reduced contrast enhancement. MRI, magnetic resonance imaging.

\section{Management}

There is no standard protocol employed in the management of spinal PNETs. Instead, most treating physicians use empirical and often kitchen-sink type approaches. In general, most centers employ surgical biopsy or resection, chemotherapy, and radiation therapy in their treatment protocol. In our present case, we employed complete removal of the intradural portion and started adjuvant radiation therapy later on as per the recommendation of Jason et al.

\section{Follow-up and Prognosis}

The prognosis in most cases of spinal PNETs appears to be poor with a median patient survival of 1 to 2 years. ${ }^{15}$ Approximately one-third of patients will exhibit cerebrospinal dissemination of their tumor. ${ }^{16}$ Metastasis to bone, liver, and cervical lymph nodes have also been reported, thus follow-up with magnetic resonance imaging and possibly fluorodeoxyglucose-positron emission tomography scanning are important. ${ }^{11}$

The present case did not show any evidence of dissemination or metastasis till first follow-up 6 weeks postoperatively. However, the patient has been referred to the radiotherapy department for further managementespecially for extraspinal residual.

\section{Conclusion}

Due to the low incidence of these tumors, the available epidemiology is likely unreliable, and there are currently no standard clinical guidelines outlining their management. In addition, the existing literature on primary spinal PNETs consists mostly of case reports with a variety of clinical presentations, management recommendations, and outcome data indicating a need for further study.

\section{References}

1 Dehner LP. Primitive neuroectodermal tumor and Ewing's sarcoma. Am J Surg Pathol 1993;17(1):1-13

2 Kosnik EJ, Bosel C, Bay J, Sayers MP. Primitive neuroectodermal tumors of the central nervous system in children. J Neurosurg 1978;48(5):741-746

3 Sevick RJ, Johns RD, Curry BJ. Primary spinal primitive neuroectodermal tumor with extraneural metastases. AJNR Am J Neuroradiol 1987;8(6):1151-1152

4 Kaspers GJ, Kamphorst W, van de Graaff M, van Alphen HA, Veerman AJ. Primary spinal epidural extraosseous Ewing's sarcoma. Cancer 1991;68(3):648-654

5 Musahl V, Rihn JA, Fumich FE, Kang JD. Sacral intraspinal extradural primitive neuroectodermal tumor. Spine J 2008;8(5):1024-1029

6 Ozdemir N, Usta G, Minoglu M, Erbay AM, Bezircioglu H, Tunakan M. Primary primitive neuroectodermal tumor of the lumbar extradural space. J Neurosurg Pediatr 2008;2(3):215-221

7 Jingyu C, Jinning S, Hui M, Hua F. Intraspinal primitive neuroectodermal tumors: Report of four cases and review of the literature. Neurol India 2009;57(5):661-668

8 Ellis JA, Rothrock RJ, Moise G, et al. Primitive neuroectodermal tumors of the spine: a comprehensive review with illustrative clinical cases. Neurosurg Focus 2011;30(1):E1

9 Schellinger KA, Propp JM, Villano JL, McCarthy BJ. Descriptive epidemiology of primary spinal cord tumors. J Neurooncol 2008; 87(2):173-179

10 Hart MN, Earle KM. Primitive neuroectodermal tumors of the brain in children. Cancer 1973;32(4):890-897 
11 Louis DN, Ohgaki H, Wiestler OD, Cavenee WK. WHO Classification of Tumours of the Central Nervous System, 4th ed. Vol. 1. Lyon, France: International Agency for Research on Cancer; 2007

12 Gessi M, Giangaspero F, Lauriola L, et al. Embryonal tumors with abundant neuropil and true rosettes: a distinctive CNS primitive neuroectodermal tumor. Am J Surg Pathol 2009; 33(2):211-217

13 Fabre E, Guillevin R, Chretien F, Le Guerinel C, Duffau H. Peripheral primitive neuroectodermal tumor of the cauda equina in an elderly patient. Case report. J Neurosurg Spine 2006;5(1):68-71

14 Hrabálek L, Kalita O, Svebisova H, et al. Dumbbell-shaped peripheral primitive neuroectodermal tumor of the spine-case report and review of the literature. J Neurooncol 2009;92(2):211-217

15 Kumar R, Reddy SJ, Wani AA, Pal L. Primary spinal primitive neuroectodermal tumor: case series and review of the literature. Pediatr Neurosurg 2007;43(1):1-6

16 Horten BC, Rubinstein LJ. Primary cerebral neuroblastoma. A clinicopathological study of 35 cases. Brain 1976;99(4):735-756 